Artigo / Article

\title{
Fatores associados à aptidão clínica para a doação de sangue - determinantes demográficos e socioeconômicos
}

\author{
Factors associated to clinical aptness for blood donation - demographic and socioeconomic \\ determinants
}

\author{
Stela Brener ${ }^{1}$ \\ Waleska T. Caiaffa ${ }^{2}$ \\ Emília Sakurai ${ }^{3}$ \\ Fernando A. Proietti ${ }^{2}$
}

\begin{abstract}
Crescimento significativo da preocupação com a segurança transfusional vem sendo observado nas últimas décadas, paralelo às alterações demográficas e sociais da população, além dos avanços técnico-cientificos, que aumentam naturalmente a demanda por transfusões de sangue. Entretanto, apesar dos investimentos na captação de doadores, seu déficit continua sendo crônico. Este trabalho teve como objetivo comparar aspectos demográficos e socioeconômicos entre indivíduos aptos, inaptos temporários e inaptos permanentes na triagem clínica para a doação de sangue, visando identificar fatores diferenciais. Constou de estudo caso-referente realizado no Hemocentro de Belo Horizonte (1994-1995), a partir de um inquérito envolvendo 3.527 candidatos à doação. Comparações das características dos candidatos aptos e inaptos foram realizadas, estratificadas por sexo. Regressão logística multinomial foi utilizada para investigar os fatores associados à aptidão para a doação. Os candidatos à doação se assemelhavam quanto ao nível socioeconômico e eram dissimilares na distribuição por sexo, faixa etária e tipo de doação. Para ambos os sexos, as seguintes características se associaram estatisticamente à aptidão: ser mais jovem (18 a 29 anos), ter união estável, estar trabalhando e não possuir automóvel. Para homens incluiu-se, ainda, ter até duas pessoas por dormitório na residência. Distintos perfis demográficos e socioeconomicos foram identificados, de acordo com o sexo e categorias de aptidão, com maior similaridade entre o perfil dos indivíduos inaptos clínicos temporários, principalmente mulheres, com o dos aptos clínicos. Portanto, justifica-se a adoção de múltiplas estratégias de captação de doadores para atingir públicos alvos especificos. Rev. bras. hematol. hemoter. 2008;30(2):108-113.
\end{abstract}

Palavras-chave: Doador de sangue; demografia; aspectos socioeconômicos; aptidão clínica.

\section{Introdução}

Nas últimas duas décadas houve crescimento considerável da preocupação com a garantia da segurança transfusional, desencadeada principalmente pelo surgimento da epidemia de AIDS. ${ }^{1}$ Paralelamente, o envelhecimento da população, a violência e os acidentes, associados aos avanços técnico-científicos na área médica, trouxeram um aumento na demanda por transfusões, nem sempre acompanhado por um incremento no número de doadores de sangue., ${ }^{2,3}$ Colaboram com esta situação políticas que adotam um maior rigor no processo de seleção de doadores e, conseqüentemente, um decréscimo no número de indivíduos que preenchem os critérios de aptidão. ${ }^{4}$

${ }^{1}$ Fundação Hemominas.

${ }^{2}$ Grupo de Pesquisa em Epidemiologia, Departamento de Medicina Preventiva e Social, Faculdade de Medicina da Universidade Federal de Minas Gerais. Belo Horizonte. Observatório Saúde Urbana e Grupo de Pesquisa em Epidemiologia Bolsa de Produtividade CNPq.

${ }^{3}$ Departamento de Estatística, Instituto de Ciências Exatas da Universidade Federal de Minas Gerais.

Correspondência: Stela Brener

Fundação Hemominas

Alameda Ezequiel Dias, 321 - Santa Efigênia

30130-110 - Belo Horizonte-MG - Brasil

Tel.: (31) 3248-4586; Fax (31) 3226-2002

E-mail: stela.brener@hemominas.mg.gov.br 
No Brasil, o problema é agravado pelos altos percentuais de inaptidão clínica e sorológica entre indivíduos que se dispõem a doar sangue, ${ }^{5}$ além dos elevados custos financeiros que envolvem a garantia da segurança transfusional, hoje em grande parte sob responsabilidade do sistema público. ${ }^{6}$ É, portanto, um dos grandes desafios dos serviços de hemoterapia a garantia do atendimento da demanda transfusional, aliando disponibilidade dos produtos sangüíneos à sua qualidade. ${ }^{1,4}$

O candidato à doação de sangue (CDS) foi caracterizado no estudo como o indivíduo que compareceu ao Hemocentro de Belo Horizonte (HBH) com a intenção de fazer uma doação. Ele poderia ser considerado apto clínico (AC) quando, após triagem clínica, fosse considerado apto a doar sangue, ou inapto clínico (IC), quando considerado não apto. Este poderia ser temporário (ICT), quando requisitos da triagem clínica não eram atendidos, mas com possibilidade de futura doação, ou permanente (ICP), cuja inaptidão para doação era considerada definitiva. ${ }^{7}$

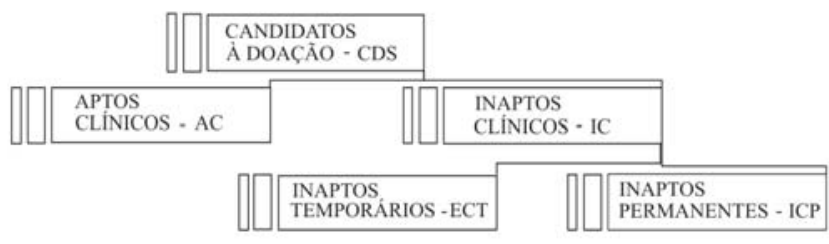

Figura 1. Caracterização dos candidatos à doação de sangue

Estudos sobre o perfil dos doadores de sangue com vistas a subsidiar ações de captação, apesar da sua importância, são pouco explorados no país onde é notável a carência de pesquisas sistematizadas na área. ${ }^{8,9,10}$ Contudo, análises comparativas têm mostrado poucas modificações substanciais em muitas das características demográficas e socioeconômicas dos candidatos à doação nos últimos anos. ${ }^{11}$

Tendo em vista o exposto, o objetivo deste estudo é caracterizar candidatos à doação de sangue em um serviço de hemoterapia e identificar fatores socioeconômicos e demográficos diferenciais associados à aptidão para a doação, de acordo com o sexo e categorias de aptidão, buscando fornecer subsídios para uma elaboração mais consubstanciada envolvendo futuras estratégias de captação de doadores de sangue.

Foram consideradas duas hipóteses: que candidatos à doação de sangue têm perfis diferenciados de acordo com o sexo e que indivíduos inaptos clínicos temporários teriam perfil mais similar ao dos indivíduos aptos clínicos, do que ao dos inaptos clínicos permanentes.

\section{Casuística e Método}

Este foi um estudo transversal, com metodologia casoreferente e parte integrante da pesquisa "Aspectos socioeconômicos, demográficos e culturais da doação de sangue em Minas Gerais", desenvolvida pela Fundação Hemominas em parceria com a Universidade Federal de Minas Gerais (UFMG).

Foi realizado em Belo Horizonte, capital do estado de Minas Gerais, Brasil, inserido na Região Metropolitana (RMBH) com 18 municípios e população de 3.436 .060 habitantes (IBGE, 1991), sendo quase $60 \%$ dos habitantes residentes na capital. ${ }^{12}$

A população do estudo foi composta por candidatos à doação de sangue (CDS) aptos clínicos (AC), inaptos clínicos temporários (ICT) e inaptos clínicos permanentes (ICP), com idade entre 18 e 60 anos de ambos os sexos, que se apresentaram para doar no HBH, no período de dezembro de 1994 a fevereiro de 1995.

Para o cálculo amostral foram considerados: a proporção estimada da menor prevalência das infecções passíveis de transmissão através da transfusão de sangue na população $(0,10 \%)$; o nível de significância de 0,05 e a precisão absoluta de $0,01 \% \cdot{ }^{13} \mathrm{~A}$ amostra final foi estimada em 2.500 candidatos AC, acrescida de $40 \%$ correspondentes ao percentual estimado de inaptidão clínica, totalizando 3.500 indivíduos.

As informações foram obtidas por meio de entrevistas face a face utilizando-se questionário contendo 199 questões, pré-codificado, planejado para o estudo e previamente testado, aplicado por entrevistadores treinados. Conforme metodologia estabelecida, testada e avaliada no estudo piloto, os CDS eram abordados de forma consecutiva após cadastramento e antes da triagem clínica, quando eram convidados a participar do estudo. A amostra final foi de 3.527 CDS, uma vez que foram excluídos 217 indivíduos devido à incompatibilização final entre os bancos de dados da amostra em estudo e o sistema de informação do HBH. Dados relativos a sexo e idade foram confrontados nos dois bancos, não sendo verificada diferença estatisticamente significante $(p<0,05)$.

A variável dependente foi aptidão clínica para a doação, categorizada em: AC, ICT e ICP e estratificada por sexo. Variáveis independentes foram agrupadas em características demográficas (por ex., idade e estado marital) e socioeconômicas (por ex., estar trabalhando, renda per capita). Aspectos clínicos ligados à inaptidão foram também pesquisados.

Análise estatística

Foram feitas análises univariadas, bivariadas e regressão logística multinomial para cada categoria de sexo do doador. A análise univariada foi utilizada para determinar distribuição das freqüências e escolha das variáveis a serem estudadas. Dado o grande número de variáveis, optamos por incluir nas tabelas apenas aquelas que se mostraram estatisticamente significantes.

A escolha das variáveis para inclusão no modelo logístico multinomial foi baseada na interpretação e relevância biológica e epidemiológica, nas estimativas dos odds 
ratios $(\mathrm{OR})$ e seu intervalo de confiança a $95 \%$, além do valor da estatística dos testes exato de Fisher e do qui-quadrado de Pearson $\left(\chi^{2}\right)$, com nível de significância de $\mathrm{p}<0,20$. Na análise multivariada, a variável dependente foi categorizada pela aptidão à doação, sendo a categoria de referência AC. Idade, categorizada entre 18 a 29 anos e 30 a 60 anos, foi sempre mantida nos modelos para ambos os sexos. As variáveis foram introduzidas em bloco, a exclusão foi manual e o critério para permanência da variável nos modelos foi o nível de significância, $\mathrm{p}<0,05 .{ }^{14}$

Foram utilizados os programas estatísticos SPSS versão 11.5 e Epi Info versão 6.4 .

Considerações éticas - $\mathrm{O}$ estudo foi aprovado pelos Comitês de Ética em Pesquisa da Hemominas, em 18/06/1998, e da UFMG, no ETCI 322/04, em 02/02/2005.

\section{Resultados}

Entre os 3.527 participantes da pesquisa, a maioria $(60,8 \%)$ era domiciliada em Belo Horizonte. A aptidão clínica para a doação de sangue foi de 56,6\% (1.996) e, entre os IC, $90,9 \%$ (140) eram ICT. Homens representavam $76,5 \%$ entre os CDS e mulheres $23,5 \%$, com idade média igual a $30 \pm 9,2$ anos e $29 \pm 8,9$ anos, respectivamente. Mais da metade dos participantes tinha entre 18 e 29 anos $(57,1 \%)$. Mulheres relataram possuir mais de oito anos de estudo $(51,2 \%)$ e homens de um a oito anos $(61,2 \%)$. Verificou-se predomínio de indivíduos que não possuíam união estável $(58,1 \%)$ (Tabela 1$)$.

A maioria dos CDS estava trabalhando $(77,1 \%)$, com maior percentual entre homens $(81,9 \%)$ do que entre mulheres $(61,2 \%)$ (Tabela 2). Constatou-se um predomínio de indivíduos inseridos no comércio de mercadorias tanto para homens $(16,1 \%)$, quanto para mulheres $(20,4 \%)$, mas com maior freqüência de profissionais da área de ensino entre estas últimas. Relativa similaridade foi observada na renda per capita, densidade demográfica no domicílio, medida pelo número de pessoas no dormitório e posse de automóvel, independente do sexo e categoria de aptidão (Tabela 2).

A aptidão clínica para doação de sangue mostrou valores limítrofes de significância estatística $(\mathrm{OR}=1,17$, IC 95\% $=1,00-1,37)$ de acordo com sexo, com mulheres apresentando 1,2 vezes a chance de serem AC quando comparadas com homens. Um comportamento diferenciado na aptidão para doação de acordo com sexo e idade também pode ser observado no Gráfico 1. Da mesma forma, a distribuição das principais causas de inaptidão apresenta diferenças marcantes entre homens e mulheres (Tabela 3).

Quando comparados aos indivíduos AC, aqueles ICP de ambos os sexos eram mais velhos (30 a 60 anos), não tinham união estável, não estavam trabalhando e apenas os homens possuíam automóvel. Indivíduos ICT do sexo masculino mais freqüentemente não tinham união estável e apresentavam em suas residências mais de duas pessoas por dormitório. Mulheres ICT relataram possuir automóvel. Cabe ressaltar que participantes $\mathrm{AC}$, de ambos os sexos, mais freqüentemente, eram mais jovens ( 18 a 29 anos), tinham união estável, estavam trabalhando e não possuíam automóvel. Para homens incluiu-se, ainda, ter em sua residência duas ou me-

Tabela 1. Distribuição proporcional das características demográficas dos candidatos à doação de sangue que compareceram ao Hemocentro de Belo Horizonte, de acordo com sexo e categoria de aptidão. Dezembro de 1994 a fevereiro de 1995

\begin{tabular}{|c|c|c|c|c|c|c|}
\hline \multirow{3}{*}{$\begin{array}{c}\text { Demográficas } \\
\text { n (\%) }\end{array}$} & \multicolumn{3}{|c|}{ Masculino $(n=2.699)$} & \multicolumn{3}{|c|}{ Feminino $(n=828)$} \\
\hline & \multirow{2}{*}{$\begin{array}{c}\text { Aptos clínicos } \\
n=1.503\end{array}$} & \multicolumn{2}{|c|}{$\begin{array}{l}\text { Inaptos clínicos } \\
\mathrm{n}=1.196\end{array}$} & \multirow{2}{*}{$\begin{array}{c}\text { Aptas clínicas } \\
n=493\end{array}$} & \multicolumn{2}{|c|}{$\begin{array}{l}\text { Inaptas clínicas } \\
n=332\end{array}$} \\
\hline & & $\begin{array}{c}\text { Temporários } \\
\mathrm{n}=1085\end{array}$ & $\begin{array}{c}\text { Permanentes } \\
n=111\end{array}$ & & $\begin{array}{c}\text { Temporárias } \\
\mathrm{n}=306\end{array}$ & $\begin{array}{c}\text { Permanentes } \\
n=29\end{array}$ \\
\hline \multicolumn{7}{|l|}{ Idade (anos) } \\
\hline $18-29$ & $817(54,4)$ & $695(64,2)$ & $51(45,9)$ & $277(56,3)$ & $159(52,3)$ & $11(37,9)$ \\
\hline $30-39$ & $459(30,6)$ & $245(22,6)$ & $30(27,0)$ & $119(24,2)$ & $80(26,4)$ & $7(24,1)$ \\
\hline $40-49$ & $181(12,1)$ & $101(9,3)$ & $24(21,6)$ & $70(14,2)$ & $54(17,8)$ & $6(20,7)$ \\
\hline $50-60$ & $44(2,9)$ & $41(3,8)$ & $6(5,4)$ & $26(5,3)$ & $10(3,3)$ & $5(17,2)$ \\
\hline \multicolumn{7}{|l|}{ Escolaridade (anos) } \\
\hline 0 & $40(2,7)$ & $33(3,1)$ & $1(0,9)$ & $6(1,2)$ & $4(1,3)$ & $1(3,4)$ \\
\hline 1 a 8 & $890(59,6)$ & $694(64,7)$ & $72(66,1)$ & $230(47,0)$ & $151(49,8)$ & $9(31,0)$ \\
\hline$>$ de 8 & $563(37,7)$ & $345(32,2)$ & $36(33,0)$ & $253(51,7)$ & $148(48,8)$ & $19(65,5)$ \\
\hline $\begin{array}{l}\text { Estado marital } \\
\text { não estável }\end{array}$ & $742(49,7)$ & $712(65,7)$ & $74(66,7)$ & $316(64,1)$ & $185(61,1)$ & $21(72,4)$ \\
\hline \multicolumn{7}{|l|}{ Religião } \\
\hline sem religião & $132(8,8)$ & $112(10,4)$ & $12(10,8)$ & $32(6,5)$ & $20(6,6)$ & $0(0,0)$ \\
\hline católica & $1.082(72,0)$ & $801(74,0)$ & $82(73,9)$ & $349(70,8)$ & $216(71,3)$ & $21(72,4)$ \\
\hline evangélica & $200(13,3)$ & $112(10,4)$ & $12(10,8)$ & $67(13,6)$ & $38(12,5)$ & $4(13,8)$ \\
\hline outras & $89(5,9)$ & $57(5,3)$ & $5(4,5)$ & $45(9,1)$ & $29(9,6)$ & $4(13,8)$ \\
\hline
\end{tabular}

Dados não informados (valor máximo igual a $0,85 \%$, dependendo da variável) foram excluídos dos cálculos das porcentagens 
Tabela 2. Distribuição proporcional das características socioeconômicas dos candidatos à doação sangue que compareceram ao Hemocentro de Belo Horizonte, de acordo com sexo e categoria de aptidão. Dezembro de 1994 a fevereiro de 1995

\begin{tabular}{|c|c|c|c|c|c|c|}
\hline \multirow{3}{*}{$\begin{array}{c}\text { Características } \\
\text { socioeconômicas } \\
\text { n (\%) }\end{array}$} & \multicolumn{3}{|c|}{ Masculino $(n=2.699)$} & \multicolumn{3}{|c|}{ Feminino $(n=828)$} \\
\hline & \multirow{2}{*}{$\begin{array}{c}\text { Aptos clínicos } \\
n=1.503\end{array}$} & \multicolumn{2}{|c|}{ Inaptos clínicos $(n=1.196)$} & \multirow{2}{*}{$\begin{array}{c}\text { Aptas clínicas } \\
n=493\end{array}$} & \multicolumn{2}{|c|}{ Inaptas clínicas $n=332$ ) } \\
\hline & & $\begin{array}{c}\text { Temporários } \\
\mathrm{n}=1085\end{array}$ & $\begin{array}{c}\text { Permanentes } \\
n=111\end{array}$ & & $\begin{array}{c}\text { Temporárias } \\
\mathrm{n}=306\end{array}$ & $\begin{array}{c}\text { Permanentes } \\
n=29\end{array}$ \\
\hline Trabalhando $^{a}$ & $1.269(84,4)$ & $869(80,2)$ & $73(65,8)$ & $309(62,7)$ & $186(61,4)$ & $12(41,4)$ \\
\hline Empregado $^{\mathrm{b}}$ & $1.030(68,5)$ & $677(62,5)$ & $50(45,0)$ & $246(49,9)$ & $139(45,9)$ & $10(34,5)$ \\
\hline $\begin{array}{l}\text { Renda per capita/s.m. } \\
\begin{array}{l}0-0,50 \\
0,51-0,80 \\
0,81-1,25 \\
1,26-5,00\end{array}\end{array}$ & $\begin{array}{l}497(33,1) \\
332(22,1) \\
407(27,1) \\
266(17,7)\end{array}$ & $\begin{array}{l}350(32,3) \\
213(19,7) \\
311(28,7) \\
209(19,3)\end{array}$ & $\begin{array}{l}38(34,2) \\
20(18,0) \\
35(31,5) \\
18(16,2)\end{array}$ & $\begin{array}{c}175(35,5) \\
93(18,9) \\
137(27,8) \\
88(17,8)\end{array}$ & $\begin{array}{l}102(33,7) \\
55(18,2) \\
80(26,4) \\
66(21,8)\end{array}$ & $\begin{array}{c}11(37,9) \\
4(13,8) \\
12(41,4) \\
2(6,9)\end{array}$ \\
\hline Não possui automóvel & $982(65,3)$ & $704(65,0)$ & $62(55,9)$ & $305(61,9)$ & $162(53,5)$ & $17(58,6)$ \\
\hline $\begin{array}{l}\text { Pessoas/dormitório } \\
\quad+2 \\
\quad 1 \text { a } 2 \\
\quad<1\end{array}$ & $\begin{array}{l}411(27,3) \\
799(53,2) \\
293(19,5)\end{array}$ & $\begin{array}{l}359(33,1) \\
522(48,2) \\
202(18,7)\end{array}$ & $\begin{array}{l}34(30,6) \\
56(50,5) \\
21(18,9)\end{array}$ & $\begin{array}{l}120(24,3) \\
269(54,6) \\
104(21,1)\end{array}$ & $\begin{array}{c}80(26,5) \\
158(52,3) \\
64(21,2)\end{array}$ & $\begin{array}{c}4(13,8) \\
16(55,2) \\
9(31,0)\end{array}$ \\
\hline
\end{tabular}

Dados não informados (valor máximo igual a $0,12 \%$, dependendo da variável) foram excluídos dos cálculos das porcentagens ${ }^{a}$ trabalho formal e informal; ${ }^{b}$ possui patrão; ${ }^{c}$ razão entre a renda familiar no mês anterior e o número de pessoas da moradia s.m. = salário mínimo $=\mathrm{R} \$ 70,00$
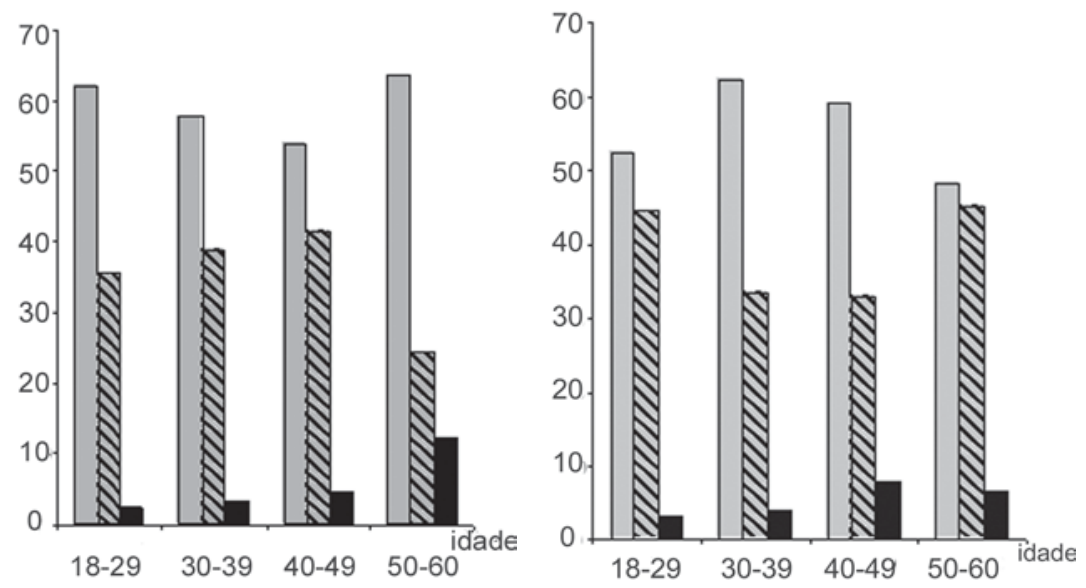

Gráfico 1. Distribuição proporcional dos candidatos à doação, por sexo e idade, de acordo com a aptidão para a doação de sangue. Belo Horizonte, dezembro de 1994 a fevereiro de 1995.

nos pessoas por dormitório. Apesar de ter apresentado valores limítrofes, a variável posse de automóvel foi considerada estatisticamente significativa (Tabela 4) e, ser empregado, foi excluída dos modelos por apresentar colinearidade com a variável estar trabalhando.

\section{Discussão}

Este é possivelmente o maior estudo já conduzido no Brasil em um único serviço de hemoterapia e uma das investigações pioneiras dos fatores associados à aptidão clínica para doação de sangue. Sua contemporaneidade é confirmada através de estudos comparativos da distribuição por sexo, idade, nível de escolaridade e tipo de doação. ${ }^{11}$
Razoável homogeneidade na faixa de renda per capita, densidade demográfica dos dormitórios e posse de automóvel sugerem que os candidatos à doação de sangue possuíam nível socioeconômico similar.

Dificuldades no incentivo à doação feminina ficaram evidenciadas pela razão de 3,3 homens/1 mulher entre CDS. ${ }^{15}$ Observou-se maior homogeneidade no seu perfil quando comparado a homens e, apesar de minoria relativa, apresentaram nível mais elevado de instrução, relatando profissões com maiores exigências de escolaridade. Programa de incentivo à doação de sangue a partir de $1986 \mathrm{em}$ escolas públicas pode ter colaborado com o maior número de profissionais do ensino entre elas. Mulheres apresentaram maior aptidão clínica quando comparadas aos homens, apesar de valores limítrofes de significância estatística, o que não ocorre quando estudamos candidatos a doadores em 2004.

Não obstante a freqüência relativa da inaptidão clínica e sua temporalidade serem similares para ambos os sexos, com relação percentual mantida também nos dados atuais, as causas, individualmente, mostraram perfil bastante diferenciado. Verificou-se que parte significativa das causas de inaptidão temporária era passível de ser transformada em aptidão para doação. A existência de indivíduos saudáveis e sensibilizados pela necessidade de doar, mas inabilitados para a doação de sangue, torna possível um impacto negativo em suas doações subseqüentes e o comprometimento da captação de potenciais doadores. ${ }^{4,16}$ 
Tabela 3. Principais causas de inaptidão clínica temporária e permanente em candidatos à doação que compareceram ao Hemocentro de Belo Horizonte, de acordo com sexo e categoria de inaptidão. Belo Horizonte, dezembro de 1994 a fevereiro de 1995

\begin{tabular}{|c|c|c|c|c|}
\hline & \multicolumn{2}{|c|}{ Inaptos clínicos masculino } & \multicolumn{2}{|c|}{ Inaptas clínicas feminina } \\
\hline & $\begin{array}{c}\text { Temporários } \\
n=1085\end{array}$ & $\begin{array}{c}\text { Permanentes } \\
n=111\end{array}$ & $\begin{array}{c}\text { Temporários } \\
n=306\end{array}$ & $\begin{array}{c}\text { Permanentes } \\
n=29\end{array}$ \\
\hline Exposição sexual & $352(32,4)$ & & $13(4,2)$ & \\
\hline Triagem sorológica positiva * & $192(17,7)$ & & $12(3,9)$ & \\
\hline Uso de medicamentos & $89(8,2)$ & & $51(16,7)$ & \\
\hline Jejum prolongado & $89(8,2)$ & & $36(11,8)$ & \\
\hline Doenças infecciosas & $70(6,5)$ & & $20(6,5)$ & \\
\hline Hipertensão & $42(3,9)$ & & $10(3,3)$ & \\
\hline Anemia ** & $18(1,7)$ & & $25(8,2)$ & \\
\hline Peso inferior a $50 \mathrm{~kg}$ & $6(0,5)$ & & $27(8,8)$ & \\
\hline Hipotensão & $16(1,5)$ & & $15(4,9)$ & \\
\hline Tratamento dentário & $22(2,0)$ & & $6(2,0)$ & \\
\hline Doença neurológica ou psiquiátrica & & $20(18,2)$ & & $9(31,0)$ \\
\hline Hipertensão arterial & & $15(13,6)$ & & $8(27,6)$ \\
\hline Alcoolismo crônico & & $23(20,9)$ & & - \\
\hline Antecedentes de hepatite & & $15(13,6)$ & & $3(10,3)$ \\
\hline Homossexualismo & & $15(13,6)$ & & $2(6,9)$ \\
\hline Uso de drogas ilegais & & $12(10,9)$ & & $1(3,4)$ \\
\hline Doenças cardiovasculares & & $3(2,7)$ & & $4(13,8)$ \\
\hline Doenças endócrinas & & $2(1,8)$ & & $1(3,4)$ \\
\hline Comp./situação risco / DST & & $2(1,8)$ & & $1(3,4)$ \\
\hline Confinamento obrigatório & & $3(2,7)$ & & - \\
\hline
\end{tabular}

* liberação posterior

** liberação posterior após tratamento

Tabela 4. Modelo final das características demográficas e socioeconômicas entre candidatos à doação sangue que compareceram ao Hemocentro de Belo Horizonte, de acordo com sexo e categoria de aptidão. Belo Horizonte, dezembro de 1994 a fevereiro de 1995

\begin{tabular}{|c|c|c|c|c|}
\hline \multirow[t]{2}{*}{ Características } & \multicolumn{2}{|c|}{$\begin{array}{c}\text { Inaptos Clínicos Masculino }(n=2699) \\
\text { OR (IC 95\%) }\end{array}$} & \multicolumn{2}{|c|}{$\begin{array}{c}\text { Inaptas Clínicas Feminina }(\mathrm{n}=828) \\
\text { OR (IC 95\%) }\end{array}$} \\
\hline & Temporários & Permanentes & Temporários & Permanentes \\
\hline Idade & & & & \\
\hline $\begin{array}{l}30-60 \\
18-29\end{array}$ & $\begin{array}{c}1,00 \\
1,04(0,86-1,26)\end{array}$ & $\begin{array}{c}1,00 \\
0,32(0,20-0,52)\end{array}$ & $\begin{array}{c}1,00 \\
0,90(0,66-1,24)\end{array}$ & $\begin{array}{c}1,00 \\
0,29(0,12-0,67)\end{array}$ \\
\hline $\begin{array}{l}\text { Estado marital } \\
\text { sim } \\
\text { não }\end{array}$ & $\begin{array}{c}1,00 \\
1,92(1,58-2,34)\end{array}$ & $\begin{array}{c}1,00 \\
3,25(1,99-5,32)\end{array}$ & $\begin{array}{c}1,00 \\
0,93(0,67-1,29)\end{array}$ & $\begin{array}{c}1,00 \\
2,77(1,11-6,90)\end{array}$ \\
\hline $\begin{array}{l}\text { Trabalhando } \\
\text { sim } \\
\text { não }\end{array}$ & $\begin{array}{c}1,00 \\
1,17(0,95-1,45)\end{array}$ & $\begin{array}{c}1,00 \\
2,84(1,85-4,36)\end{array}$ & $\begin{array}{c}1,00 \\
1,07(0,79-1,44)\end{array}$ & $\begin{array}{c}1,00 \\
3,04(1,38-6,68)\end{array}$ \\
\hline $\begin{array}{l}\text { Posse de automóvel } \\
\text { sim } \\
\text { não }\end{array}$ & $\begin{array}{c}1,00 \\
0,99(0,84-1,18)\end{array}$ & $\begin{array}{c}1,00 \\
0,65(0,43-0,98)\end{array}$ & $\begin{array}{c}1,00 \\
0,72(0,54-0,96)\end{array}$ & $\begin{array}{c}1,00 \\
0,87(0,41-1,89)\end{array}$ \\
\hline $\begin{array}{l}\text { Pessoa/dormitório } \\
\quad<1 \\
1 \text { a } 2 \\
+2 \\
\end{array}$ & $\begin{array}{c}1,00 \\
1,04(0,84-1,30) \\
1,42(1,12-1,80) \\
\end{array}$ & $\begin{array}{c}1,00 \\
1,26(0,74-2,16) \\
1,75(0,98-3,18)\end{array}$ & $\begin{array}{c}1,00 \\
0,93(0,64-1,34) \\
1,16(0,78-1,79)\end{array}$ & $\begin{array}{c}1,00 \\
0,72(0,30-1,72) \\
0,39(0,11-1,36)\end{array}$ \\
\hline
\end{tabular}

Categoria de referência = aptos clínicos; OR = Odds Ratio; IC 95\% = Intervalo de Confiança a 95\%

Evidências de maior similaridade entre perfis de indivíduos ICT e AC, principalmente mulheres, demonstra a necessidade de revisão de critérios e estratégias relativas à inaptidão temporária, com adoção de enfoque de atenção especial aos indivíduos ICT voltado principalmente para seu retorno à doação. ${ }^{17,18} \mathrm{~A}$ existência de potenciais doadores entre mulheres e indivíduos de ambos os sexos, com idade superior a 30 anos e com perfil adequado à doação, torna fundamental a prática de múltiplas estratégias de captação de doadores, aliadas ao seu monitoramento sistemático. 


\begin{abstract}
Over the last decades, growing concern regarding transfusional safety has been observed, parallel to demographic and social changes as well as technological advances and increasing demand for blood transfusions. However, despite investment to increase the number of blood donors, there is a chronic shortage of blood. The aim of this study was to compare demographic and social-economic characteristics comparing individuals that were considered eligible with temporarily and permanent non-eligible blood donors. A casecomparison study was carried out at the Blood Donation Center (Hemocentro) of Belo Horizonte from a survey involving 3,527 candidates for blood donation. Comparisons stratified by gender were made for all characteristics. Multinomial logistic regression was used to verify the association between the variables and eligibility for blood donation. Candidates for blood donation were similar according to the socio-economic level and dissimilar according to gender, age and type of donation. For both genders, the following characteristics were statistically associated in respect to eligibility for blood donation: to be young (18 to 29 years), to have a stable relationship and employment and not to own an automobile. For men, the presence of less than two people per bedroom of their residence was also statistically significant. Distinct demographic and social-economic profiles were identified in relation to gender and eligibility category. Similar profiles were found between temporarily non-eligible and eligible candidates, especially among women. Therefore, adopting multiple strategies in blood donor recruitment is justified in order to make contact with the different groups. Rev. bras. hematol. hemoter. 2008;30(2):108-113.
\end{abstract}

Key words: Blood donors; demography; socioeconomic determinants; eligibility for blood donation.

\section{Agradecimentos}

O projeto "Aspectos socioeconômicos, demográficos e culturais da doação de sangue em Minas Gerais" foi financiado pela Fundação de Amparo à Pesquisa do Estado de Minas Gerais (Fapemig), processo $n^{\circ}$ CDS 1280/90-83.

Agradecemos à Dra. Angela Giviziez pela idealização do projeto, aos colegas Moema Fígoli, Roberto Nascimento, Rômulo de Souza e Stephen McCracken, pela parceria, è̀ Hemominas e ao Grupo de Pesquisa em Epidemiologia, pelo apoio na realização deste trabalho.

\section{Referências Bibliográficas}

1. Federação Internacional da Cruz Vermelha, Sociedades do Crescente Vermelho. Fazendo a diferença - Captando doadores de sangue voluntários, não remunerados. 2002.

2. Rodrigues RN, McCraken S, Souza RP, Giviziez AGN, Brener S. Aspectos sociodemográficos e culturais da doação de sangue em Belo Horizonte. In: VII Seminário sobre Economia Mineira, Diamantina. 1995;513-31.

3. Ministério da Saúde. Coordenação Nacional de Doenças Sexualmente Transmissíveis e Aids. Captação de doadores de sangue. Série TELELAB. Brasília, DF, 2001; 65p.
4. Beijar JE. The donor/demand dilemma. Transfusion Medicine Update. Boletim "on line" Pennsylvania: Institute for Transfusion Medicine Pittsburgh; 1996.

5. Ministério da Saúde. Agência Nacional de Vigilância Sanitária. Serviços de hemoterapia: relatórios de produção. Brasília, DF, 2002 [Internet site]. Disponível:http://www.anvisa.gov.br/sangue/ hemoterapia/relatórios_produção/inde x.htm. Acessado em 05/02/ 2005 .

6. Ministério da Saúde. Portaria MS 1.284 de 29 de junho de 2004. Altera os valores da tabela SAI/SUS, referente a remuneração de procedimentos hemoterápicos. Pub Diário Oficial da União - Seção $1,214-215, N^{o} 125(01 / 07 / 2004)$.

7. Ministério da Saúde. Coordenação Nacional de Doenças Sexualmente Transmissíveis e Aids. Triagem clínica de doadores de sangue. Série TELELAB. Brasília, DF; 2001;66p.

8 .Ministério da Saúde. Agência Nacional de Vigilância Sanitária. Programa Nacional de Doação voluntária de Sangue, Brasília, DF, 2002 [Internet site]. Disponível em: http://www.anvisa.gov.br. Acessado em 06/2003.

9. Gutiérrez MG, Tejada ES, Cruz JR. Estúdio de factores socioculturales relacionados com la doaçión voluntária de sangre em las Américas. Rev Panam Salud Pública 2003; Edição especial 13(2/3):85-90.

10. Organizacion Mundial de la Salud (OMS), Organizacion Panamericana de la Salud (OPAS). Estúdio de los aspectos sócioculturales relacionados com la donación de sangre em América. Outubro 2000.

11. Ministério da Saúde. Agência Nacional de Vigilância Sanitária. Universidade Federal do Rio de Janeiro. Centro de Tecnologia e Ciências. Projeto Perfil do Doador de Sangue Brasileiro. 2004.

12. Instituto Brasileiro de Geografia e Estatística. [Internet site]. Disponível em: http://www.ibge.gov.br. Acessado em: outubro/2004.

13. World Health Organization (OMS). Epidemiological and Statistical Methodology Unit. Sample size determination: a user's manual. Geneva, 1986. 52p.

14. Hosmer DW, Lemeshow S. Applied Logistic Regreesion. $2^{\text {nd }}$ ed. A Wiley-Intercience Publication. John Wiley \& Sons, Inc.2000.

15. Bowlware LE, Ratner LE, Ness PM, Cooper LA, Campbell-Lee TA, Powe NR. The contribution of sociodemographic, medical, and attitudinal factors to blood donation among the general public. Transfusion. 2002;42(6):669-78.

16. Halperin D, Baetens J, Newman B. The effect of short-term, temporary deferral on future blood donation. Transfusion. 1998; $38(2): 181-3$.

17. Davey RA. The "safe" blood donor and the national blood supply: is there a new interface? [editorial]. Transfusion. 1998;38:323-6.

18. Simon LS. Where have all the donors gone? A personal reflection on the crisis in American's volunteer blood program. Transfusion. 2003;43(2):273-9.

Avaliação: Editor e dois revisores externos

Conflito de interesse: não declarado

Recebido: 16/01/2007

Aceito: 13/08/2007 